\title{
IdeAs
}

Idées d'Amériques

$17 \mid 2021$

Villes et culture dans les Amériques

\section{Culture-s matter-s. Habitants-artistes, institutions et gentrification : le cas d'un quartier d'immigration mexicain de Chicago}

Culture-s matter-s. Local Artists, institutions and gentrification: the case of a Mexican migrant neighborhood in Chicago

Culture-s matter-s. Artistas locales, instituciones y gentrificación: el caso de un barrio migrante mexicano de Chicago

\section{Aline Hémond}

\section{OpenEdition}

\section{Journals}

Édition électronique

URL : https://journals.openedition.org/ideas/11099

DOI : 10.4000/ideas. 11099

ISSN : $1950-5701$

Éditeur

Institut des Amériques

Référence électronique

Aline Hémond, «Culture-s matter-s. Habitants-artistes, institutions et gentrification : le cas d'un quartier d'immigration mexicain de Chicago », IdeAs [En ligne], 17 | 2021, mis en ligne le 01 mars 2021, consulté le 04 juin 2021. URL : http://journals.openedition.org/ideas/11099 ; DOI : https://doi.org/ 10.4000/ideas.11099

Ce document a été généré automatiquement le 4 juin 2021

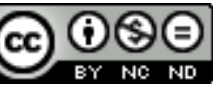

IdeAs - Idées d'Amériques est mis à disposition selon les termes de la licence Creative Commons Attribution - Pas d'Utilisation Commerciale - Pas de Modification 4.0 International. 


\title{
Culture-s matter-s. Habitants-artistes, institutions et gentrification : le cas d'un quartier d'immigration mexicain de Chicago
}

\author{
Culture-s matter-s. Local Artists, institutions and gentrification: the case of a \\ Mexican migrant neighborhood in Chicago \\ Culture-s matter-s. Artistas locales, instituciones y gentrificación: el caso de un \\ barrio migrante mexicano de Chicago
}

Aline Hémond

\section{Introduction}

1 Ce texte ${ }^{1}$ propose de s'intéresser à un quartier d'accueil des migrants mexicains dans la ville de Chicago - Pilsen - qui abrite nombre d'artistes mexicains et latino-américains, des ateliers, des galeries, des boutiques artisanales et un musée national d'art mexicain. Mon propos est de démontrer qu'il existe une tension entre, d'une part, les pratiques culturelles des artistes locaux et, d'autre part, les politiques culturelles et de rénovation urbaine qui visent à assurer l'attractivité territoriale métropolitaine. En reprenant l'optique «par le bas» développée par De Certeau (1980), ma perspective d'analyse se centrera tout d'abord sur les pratiques communautaires des artistes immigrants, en particulier dans l'art social muraliste ${ }^{2}$. Ces images fondent le collectif migrant sur le territoire d'arrivée et le confortent dans sa mise en capacité d'agir pour la reconnaissance de ses droits dans la ville (Lefebvre H., 1967).

2 Je relierai ensuite les caractéristiques de ce que l'on pourrait appeler un «village culturel » au processus accéléré de gentrification qui touche le district. Devenu très à la mode du fait de sa rénovation post-industrielle, Pilsen attire nouveaux habitants et tourisme des quartiers dits ethniques ${ }^{3}$. Cette dynamique de gentrification organise 
simultanément la relégation des populations mexicaines les plus précaires et la marchandisation du patrimoine culturel migrant.

3 Ces situations sont caractéristiques de la grande ville états-unienne, à l'organisation politique et administrative qui racialise les populations sur un territoire. Elles appellent une analyse plus approfondie de la dimension intersectionnelle entre race et classe dans les processus de gentrification ainsi que des enjeux de droit à la ville pour les populations racisées vulnérables ${ }^{4}$.

4 A cette fin, en interrogeant tour à tour la littérature sur la gentrification dite « de la demande $»^{5}$ et celle dite "de l'offre $»^{6}$, je me centrerai sur les points suivants : quel est l'impact du community mural movement à Pilsen sur l'attractivité des lieux? Qu'en est-il de la politique de la municipalité vis-à-vis des artistes et cultures urbaines ethniques en lien avec la revitalisation urbaine ? Comment les artistes migrants provoquent-ils la gentrification alors même qu'ils la combattent? La gentrification est-elle socialement plus acceptable quand les gentrifieurs procèdent en partie de la communauté immigrante ?7 Pour ce faire, je privilégierai une approche ethnographique des différents groupes d'acteurs sociaux et artistiques et un point de vue anthropologique emic $^{8}$.

\section{Fabriquer des « lieux en commun » par l'espace public : le mouvement pour la paix et le community mural movement à Pilsen}

5 Ancien quartier industriel proche de grandes fabriques et d'un nœud ferroviaire, le district de Pilsen dans le Lower West Side (carte 1), accueille tout d'abord la main d'œuvre tchèque à la fin du xix siècle.

6 Celle-ci quitte le quartier suite à l'arrivée d'une première vague de migrants mexicains dans les années 1920. C'est à partir du milieu des années 1960, puis dans les années 1980 mais surtout depuis 1995, que des flux successifs vont consolider la concentration mexicaine dans le Lower West Side (Arredondo G., 2008 ; EPA, 2013) (carte 2). 


\section{Carte 1 - Chicago, Lower West Side}

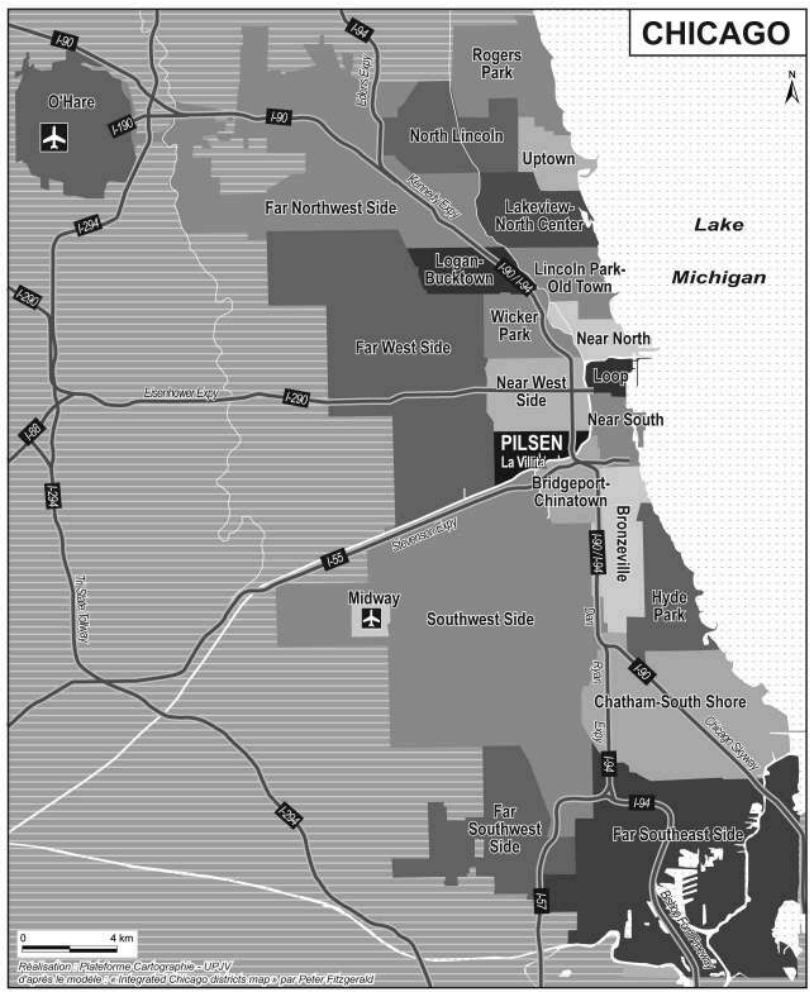

«Integrated Chicago districts map » - Plateforme Cartographie UPJV d'après modèle de Peter Fitzgerald. File : Integrated Chicago districts map.svg.

Sous licence GFDL via Wilimedia Commons.

\section{Carte 2 - District de Pilsen, Lower West Side}

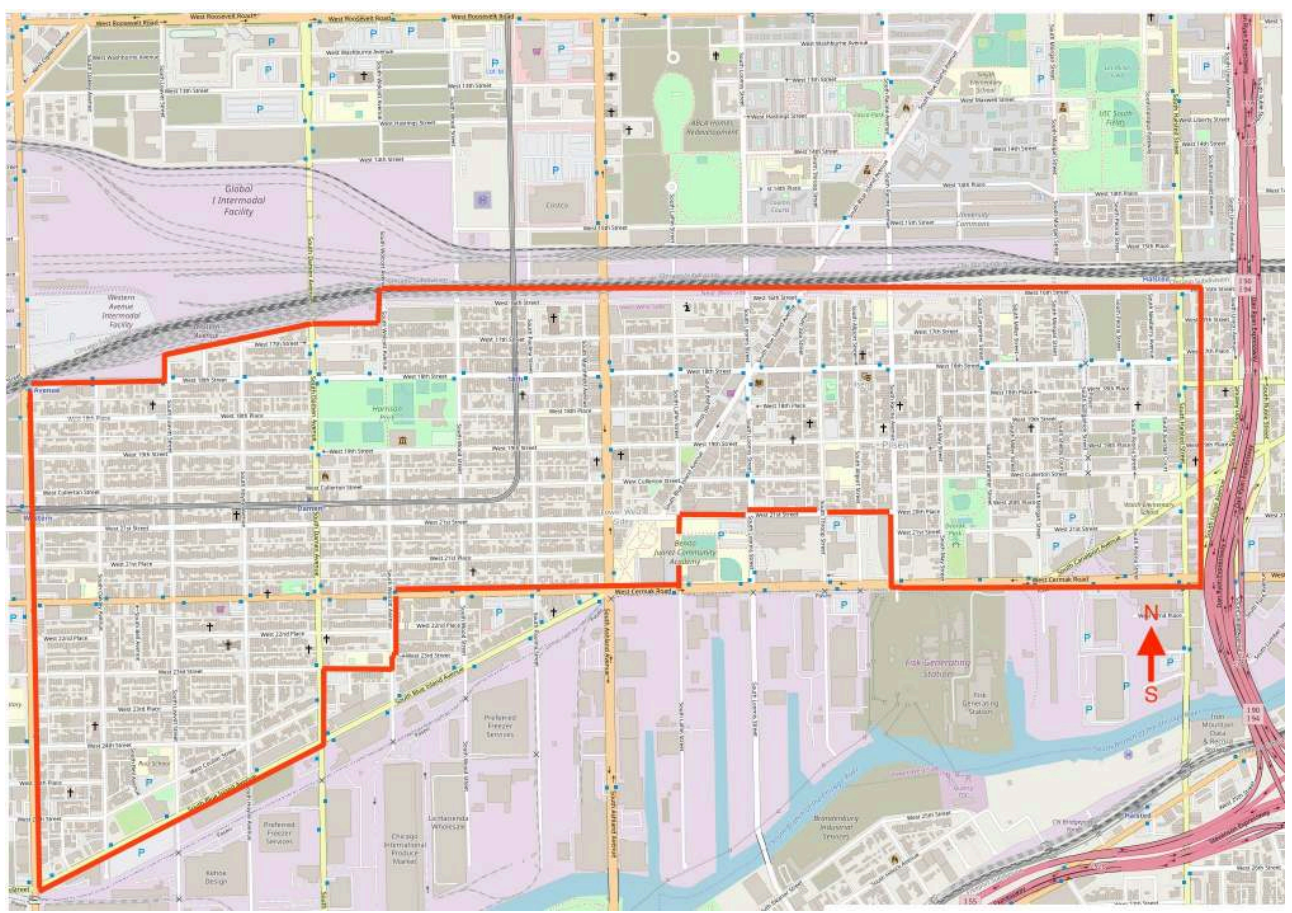

Secteur historique souligné en rouge.

https://csagsi.org/chicagos-pilsen-neighborhood/ 
7 Autre étape importante de l'ancrage mexicain dans cette partie de la ville, la constitution du mouvement de revendication chicano. A la fin des années 1960, des protestations éclatent dans les ghettos noirs et latinos du West side (Abu-Lughod J., 2007 : 12). Les travailleurs mexicains immigrés descendent dans la rue pour réclamer des droits syndicaux et salariaux, au logement et à l'éducation. Ils sont inspirés par les mobilisations organisées en Californie par le dirigeant syndical César Chávez avec les journaliers agricoles mexicains. En regroupant des centaines d'organisations communautaires unies contre la discrimination. El movimiento (ou mouvement chicano) ${ }^{9}$ devient une force de changement social dans tous les Etats-Unis. Les rues de Pilsen deviennent le théâtre de différentes mobilisations qui contribuent à faire de ce quartier (barrio) un lieu revendiqué de la visibilisation culturelle et politique de la communauté immigrante mexicaine (illustration 1$)^{10}$.

Illustration 1 : Latinismo and Chicanismo in Late 1960s Chicago.

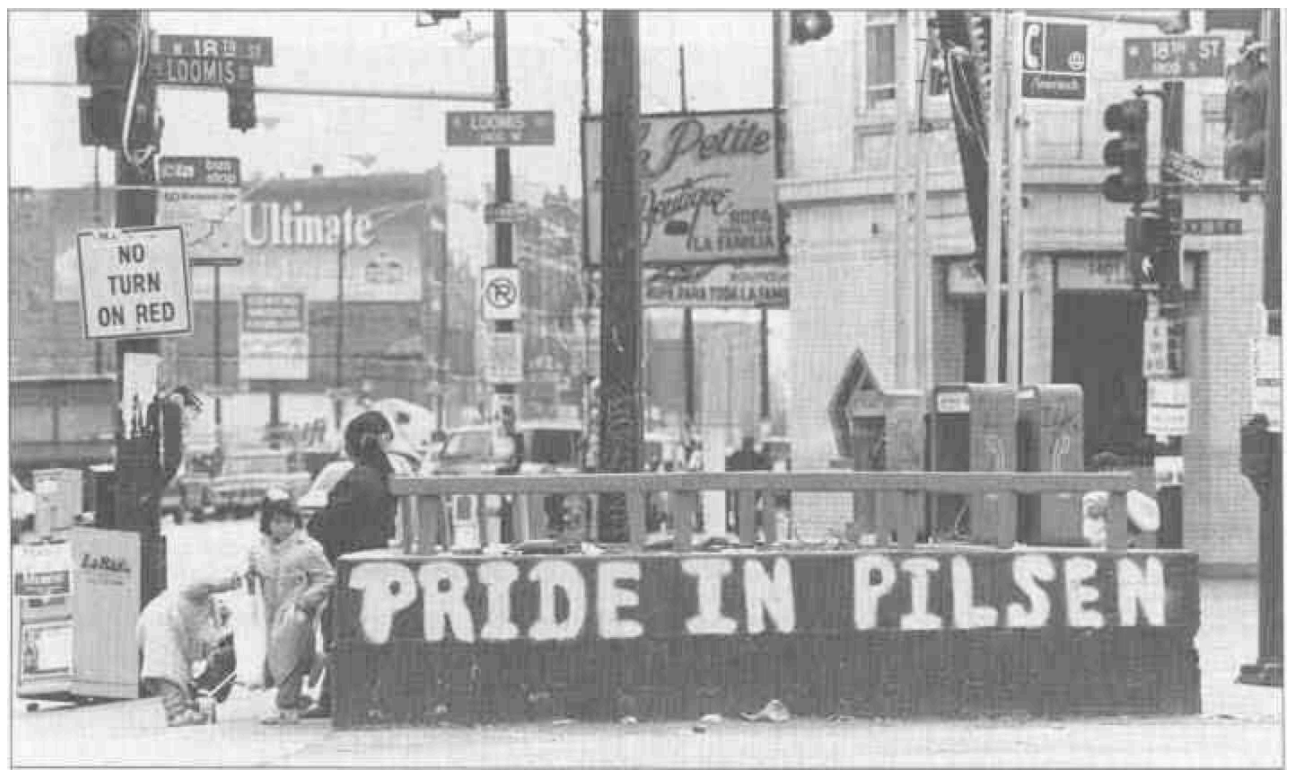

http://www.elbeisman.com/revista/post/blowouts-latinismo-and-chicanismo-in-late-1960s-chicago

"Aqui se ve la raza, aquí se ve mi gente» («Ici on voit mon peuple, on voit les miens ») estiment nombre de mes interlocuteurs ${ }^{11}$. Ces « lieux en commun », ces espaces où «la rue est à nous ${ }^{12}$ permettent de se compter en masse, de se donner à voir. Du point de vue de ses habitants, l'aspect de congrégation, de solidarité et de fierté prime sur l'aspect de ségrégation, renversant ainsi le stigmate. Des marches de mères de famille et d'étudiants ont également lieu dans la deuxième moitié des années 1970 pour réclamer un service de voirie correcte ainsi que des écoles bilingues pour une meilleure éducation. Ces actions ont abouti à la création de la Benito Juárez High School en 1977. Mais le véritable rite civique de fondation aux yeux de la communauté a lieu en 1979 avec la réalisation collective des peintures murales ornant les murs de l'école, appelées «A la esperanza $»^{13}$.

9 Ce dernier exemple montre que le répertoire de l'action collective associe les rassemblements et mobilisations dans l'espace public à d'autres formes d'activisme, notamment le community-based mural movement - l'art muraliste collectif, politiquement et socialement orienté. C'est tout d'abord la communauté africaine-américaine qui 
montre sa présence au sud de l'agglomération, en exécutant des fresques du type Wall of respect $^{14}$. Ces portraits d'hommes et de femmes noires célèbres sur les murs de la ville constituent des marqueurs visuels pour les groupes sociaux racisés qui vivent dans des quartiers de relégation. La communauté mexicaine de Pilsen va s'emparer de ces modalités de visibilisation dans les années 1970 , puis $1980^{15}$, dans les rues comme dans les espaces collectifs (écoles, centres sociaux ou salles paroissiales catholiques). Ses membres - de nombreux artistes confirmés qui ont alors un atelier à Pilsen ou à Little Village -, sont porteurs d'une double tradition picturale, politique et sociale. Celle tout d'abord du grand courant du muralismo mexicain post-révolutionnaire ${ }^{16}$ qui fabrique pour le peuple le palimpseste historique de la nation sur les murs des palais politiques mexicains. Mais aussi la gravure satirique et politique anti-gouvernementale (Taller de gráfica popular). Le community mural movement vient s'ajouter à cet héritage plastique et politique, en théorisant le "faire-ensemble». Réaliser collectivement des histoires visuelles permet aux groupes minorisés de gagner en capacité d'agir (empowerment) et en auto-organisation (Rogovin M. et al., 1975 ; Kwon M., 2002).

Au-delà des conflits et de la compétition pour l'espace urbain, on trouve donc des exemples de collaboration entre communauté africaine-américaine, immigrants mexicains, mouvement chicano et artistes blancs. Ils sont caractéristiques de la manière dont les pratiques artistiques - indissociables de l'esprit activiste d'une époque (mouvement des droits civiques puis de la paix pour le Vietnam) - ont imprégné la circulation d'idées et les actions collectives (illustration $2 a$ et $b)^{17}$. Cette insistance sur l'utilisation des arts visuels est également à analyser comme un ensemble de tactiques propres à beaucoup de groupes, migrants, minorisés ou marginalisés, pour « devenir visibles » dans l'espace urbain ${ }^{18}$. Dans le cas de Pilsen, les arts muralistes peuvent être compris comme des "arts de la visibilisation» qui fondent les migrants comme communauté politique, dans une geste visuelle revendiquant à la fois le «droit à la ville» (Lefebvre H., $1967^{19}$ ) et le droit au travail. On trouve par exemple une iconographie murale (illustration 3) représentant Emiliano Zapata, héros de la révolution mexicaine et artisan de la distribution de terres aux paysans. Son slogan : "la tierra es de quien la trabaja » lui permet de figurer comme emblème de la lutte des Mexicains immigrés pour leurs droits migratoires et contre la gentrification. 
Illustration 2a : People'Art Movment - Prevent Word War III Mural (début années 1980). Restauration de 2016 par Marco Raya. (2399 W 18 th St.)

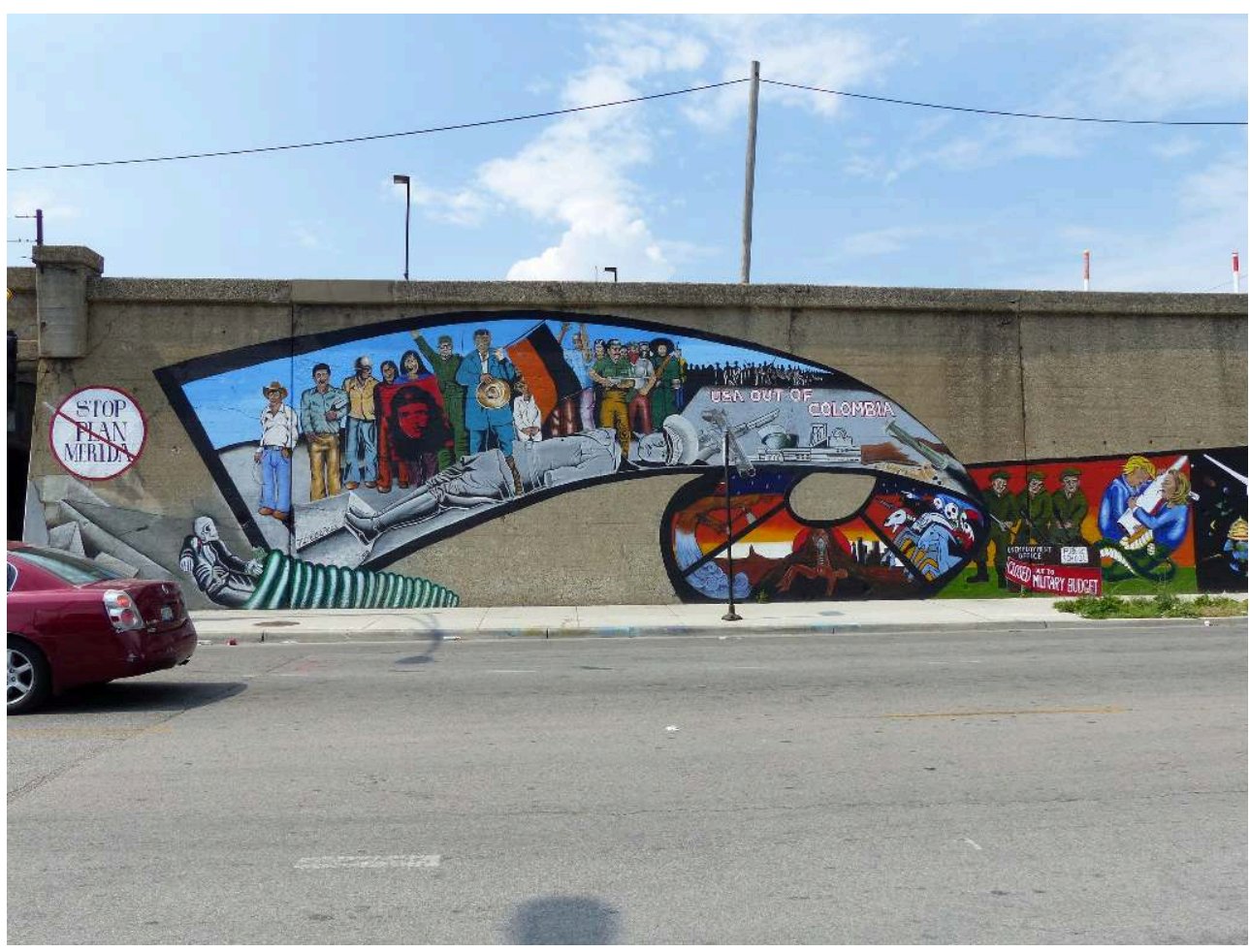

People'Art Movment - Prevent Word War III Mural (début années 1980). Restauration de 2016 par Marco Raya (2399 W 18 th St.).

(c) Hémond, 2016 
Illustration 2b : Détail Illustration précédente - People'Art Movment - Prevent Word War III Mural (début années 1980). Restauration de 2016 par Marco Raya - Inscriptions « No to gentrification, Affordable Housing in Pilsen » réalisées par Amanda Mudrovich. (2399 West 18 th St.)

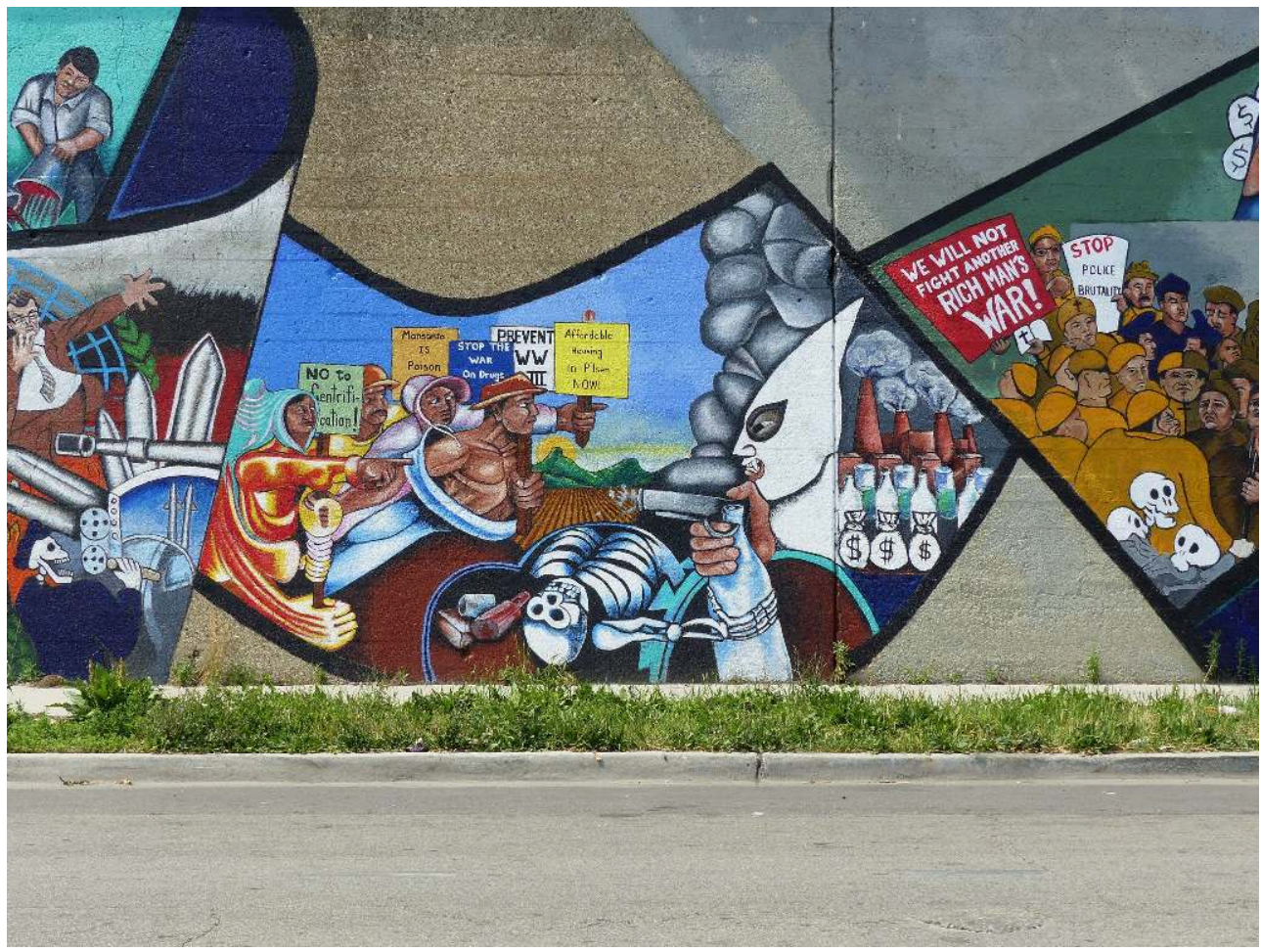

Détail Illustration précédente - People'Art Movment - Prevent Word War III Mural (début années 1980) Restauration de 2016 par Marco Raya - Inscriptions « No to gentrification, Affordable Housing in Pilsen » réalisées par Amanda Mudrovich. (2399 West 18 th St.)

(C) Hémond, 2016 
Illustration 3 : Mural représentant E. Zapata. Inscription effacée « Stop the gentification in Pilsen (Zapata vive) ». (1439 South Bishop St.)

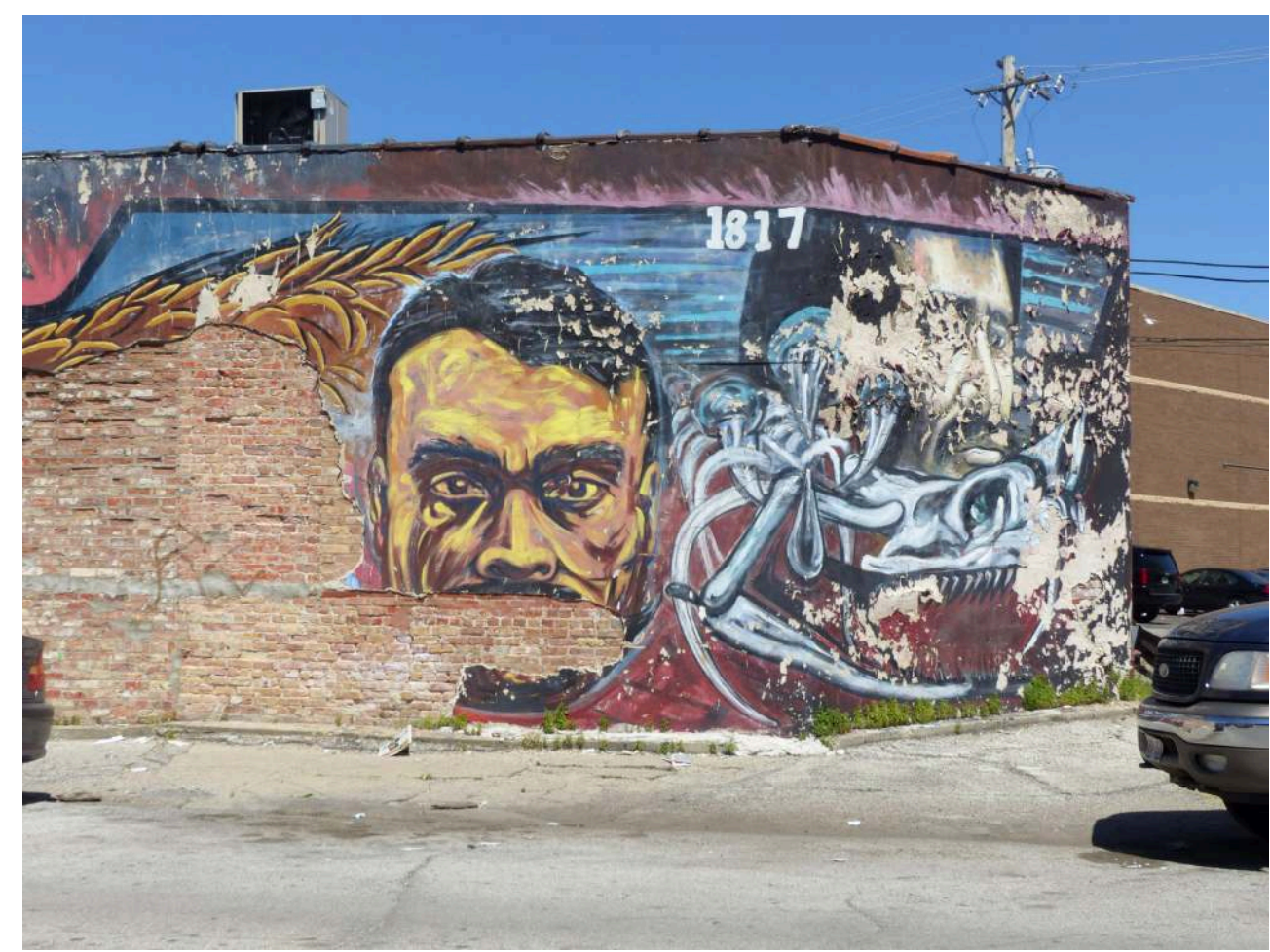

Mural représentant E. Zapata. Inscription effacée « Stop the gentification in Pilsen (Zapata vive) » (1439 South Bishop St.).

(c) Hémond, 2014

11 Si nous revenons maintenant sur la récente exposition ${ }^{20}$ commémorant le mural «A 40 años de La Esperanza» au Musée National d'Art Mexicain (NMMA), leurs curateurs ont insisté sur ce qui a été appelé « l'esprit » de la Dieciocho ou le 18th Street artwork ${ }^{21}$. Cette artère principale du district de Pilsen se couvre à partir des années 1980 de peintures murales qui donnent de la dignité et de la visibilité à l'histoire et à la culture mexicaine en terre d'arrivée, confortant ainsi une identité locationale (Kwon M., 2002). Les places, carrefours et rues adjacentes se couvrent d'un palimpseste graphique et d'un mobilier urbain spécifique (plaques de métal ornées de motifs aztèques; noms de rue bilingues), aidées en cela par la municipalité qui cherche à faire naître un tourisme des quartiers ethniques de la ville. Des centres communautaires s'ouvrent, tel Casa Aztlán fondée en 1970, sise au 1831 South Racine Avenue, recouverte de fresques de héros de l'histoire mexicaine, réalisées par Salvador Vega et Roberto Valadez. Ce centre culturel et social a contribué de manière fondamentale à l'organisation des migrants. Il est irrigué par l'esprit de protestation politique issu à la fois du combat pour les droits civiques, puis du mouvement étudiant mexicain de $1968^{22}$. Il a été moteur pour le mouvement chicano à Chicago Ses membres s'investissent également dans les grandes marches latinas du printemps 2006 pour protester contre la réforme législative criminalisant les migrants sans-papiers ${ }^{23}$.

12 Les trois dernières décennies ont vu l'exécution de murs peints dont les registres figuratifs, les commanditaires et les exécutants sont pluriels (illustration 4 à 7). Ils confortent Pilsen comme le " haut-lieu » de la haute-culture mexicaine. Y contribuent également son art de vivre : cuisine populaire ou gastronomique, fêtes et cérémonies 
(fête des morts, fête de la Vierge de Guadalupe, par exemple). L'aspect culturel mexicain s'est également développé avec de nombreux artistes, des galeries et un Musée National d'Art mexicain $(\mathrm{NMMA})^{24}$. Ce dernier espace a contribué à patrimonialiser la haute culture mexicaine et à l'institutionnaliser. Un auditorium accessible aux réunions de la communauté mexicaine et mexicaine-américaine permet de pallier l'absence de tiers-lieux depuis que Casa Aztlán a dû fermer ses portes en 2013, expulsé de son bâtiment historique du fait de la gentrification touchant Pilsen ${ }^{25}$.

Illustration 4 : Mural «Si se puede ». (Cérémonie de remise de diplôme)

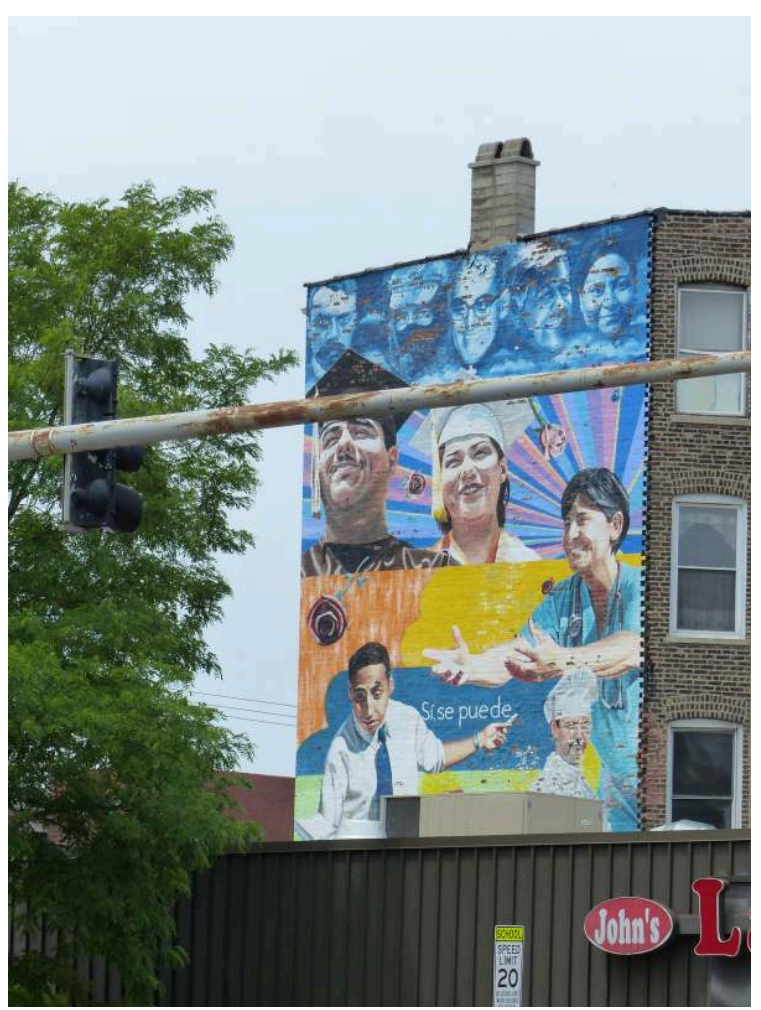

Des artistes blancs (comme Jeff Zimmermann, né à Chicago) ont réalisé un triptyque mural près de l'église de St Pius V diffusant des idées citoyennes et sociales. Sur le panneau du fond, le slogan « Si, se puede » (oui, on peut) avec des portraits de résidents qui ont réalisé leur rêve américain d'amélioration économique et sociales (angle South Ashland Av. et West 18th PI).

(c) Hémond 
Illustration 5 : Mural de Selena (1971-1995), grande idole de la chanson texmex, dont la silhouette est apparue sur les murs d'une boucherie de Pilsen (Carnicería Maribel) en mai 2019 (angle W Cermak Road et S Wood St, Pilsen).

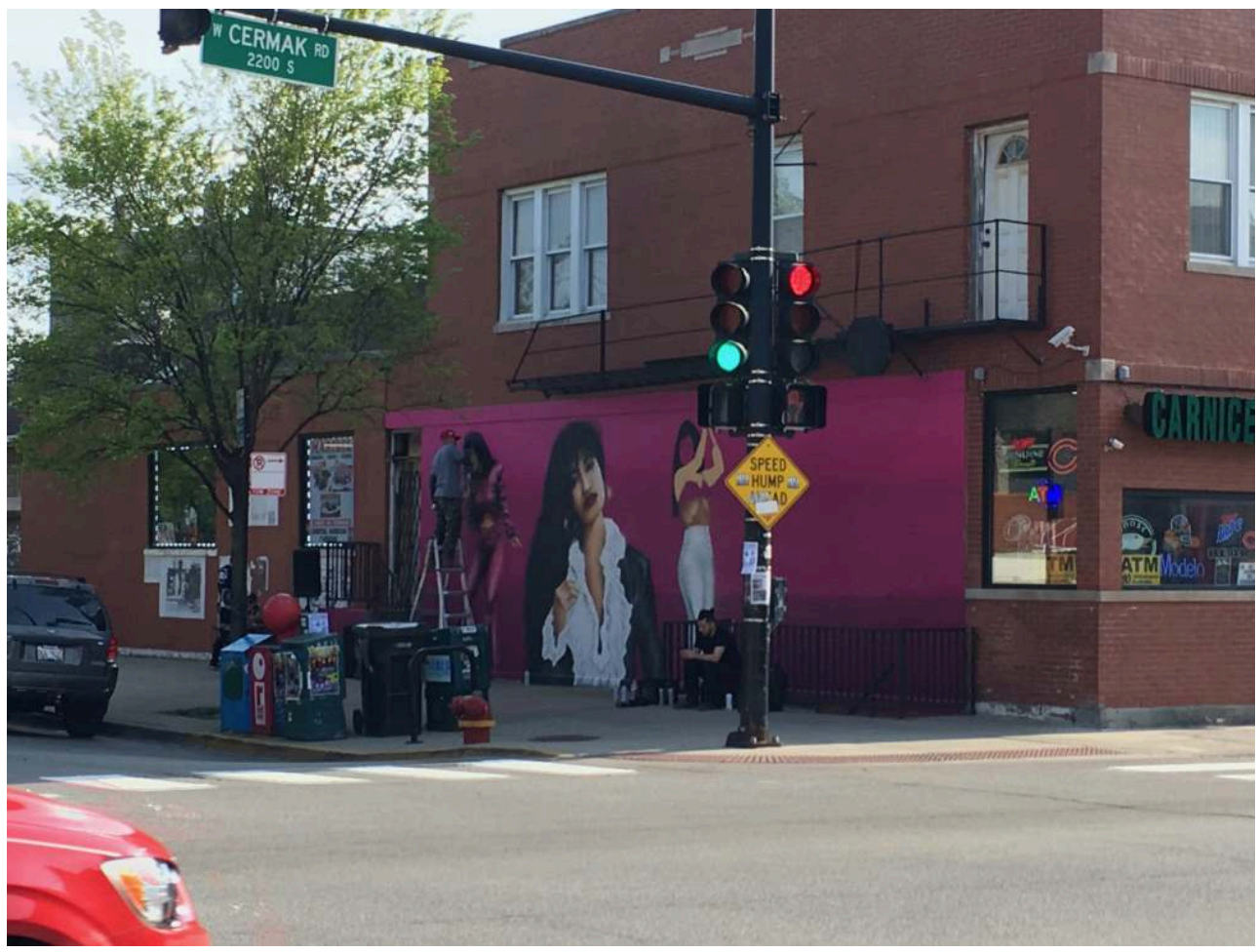

Mural de Selena (1971-1995), grande idole de la chanson texmex, dont la silhouette est apparue sur les murs d'une boucherie de Pilsen (Carnicería Maribel) en mai 2019 (angle W Cermak Road et S Wood St Pilsen).

(C) Hémond 2019 
Illustration 6 : Mural de l'agriculture, commandité par une fabrique de tortillas (galettes de maïs).

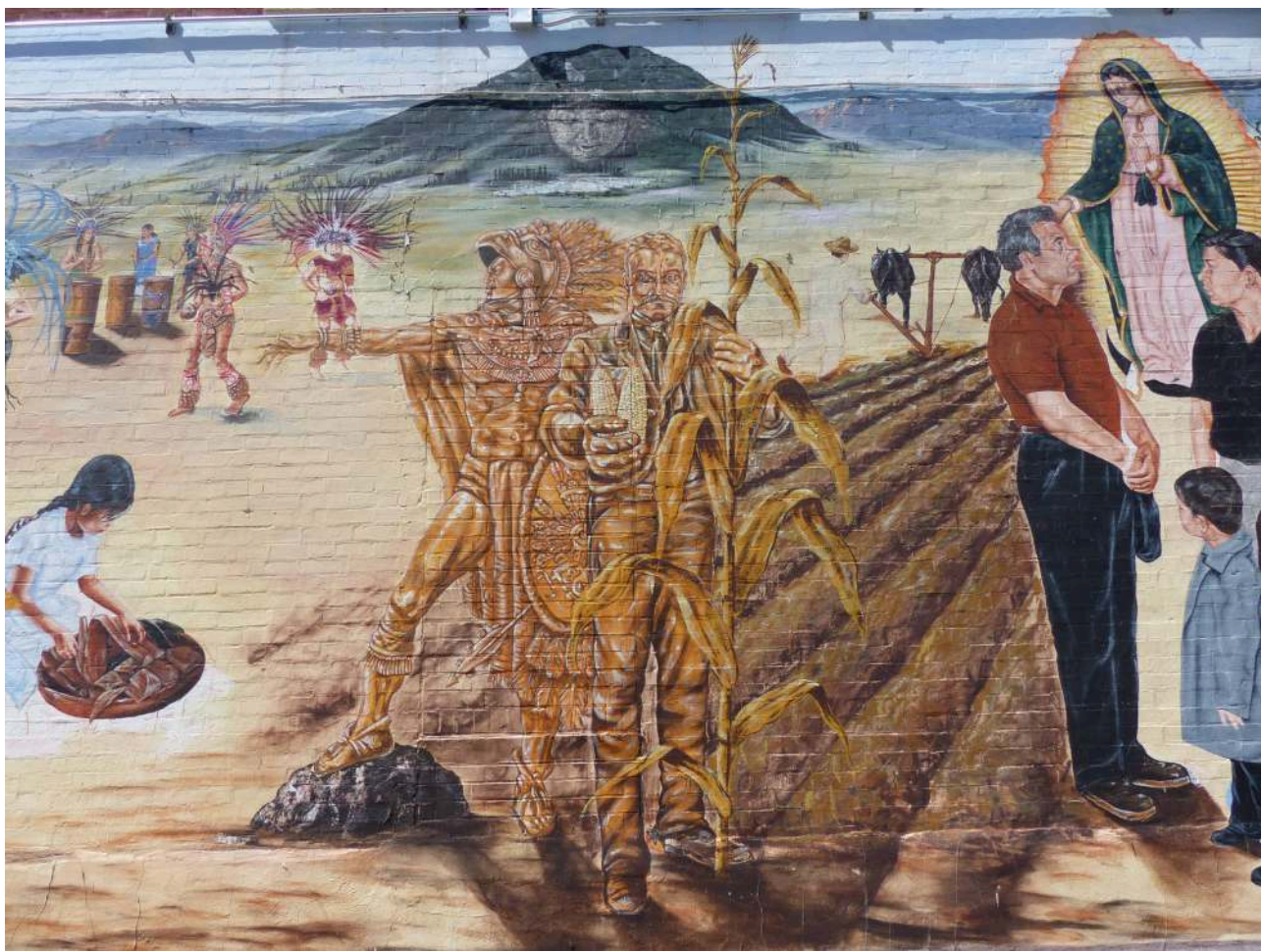

Détail représentant Zapata et le Dieu du maïs (1480 West Cullerton St., Pilsen).

(c) Hémond, 2014 


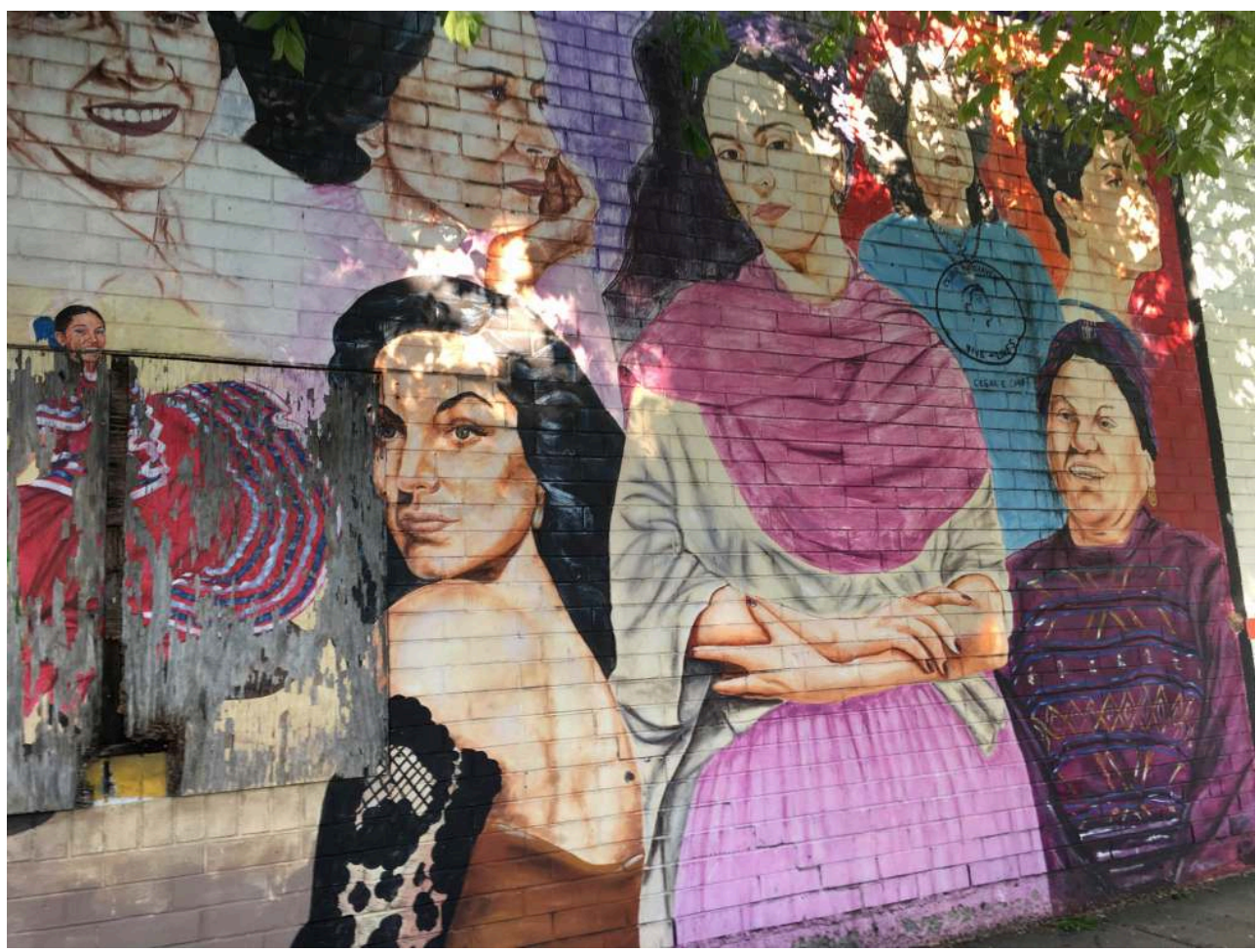

Mural sur des femmes mexicaines célèbres - détail : Adelitas, Maria Felix, Frida Kahlo, Rigoberta Menchú Tum (maya guatémaltèque) (Angle South Wood et $18^{\text {th }}$ St., Pilsen).

(c) Hémond, 2019

\section{Politiques publiques de la ville et gentrification : des effets complexes}

13 Marquée par la désindustrialisation, le chômage et le déclin démographique dans les années 1980, comme toute la Rust Belt, la ville de Chicago va se réinventer en métropole des arts et en pôle attractif du tourisme à l'instigation des politiques municipales. Avec le constat que les arts et la culture stimulent la croissance, la mutation vers l'économie culturelle et touristique ${ }^{26}$ va remodeler la ville, en lien avec l'immobilier comme valeur refuge pour les capitaux. De grands travaux de rénovation urbaine sont menés dès la fin du mandat du maire Richard J. Daley (1955-1976), poursuivis par Harold Washington (1983-1987) ${ }^{27}$ et prennent une ampleur toute particulière avec Daley fils (1989-2011). Ce dernier cherche en particulier à redynamiser le centre-ville (The Loop, Downtown) sur sa partie orientale. Sous ses mandats renouvelés, les friches ferroviaires situées le long du lac Michigan sont réaménagées avec la création du Millenium Park (2004) et du parc d'attraction du Navy Pier. Pour ce faire, des politiques de financement public-privé (public-private partnership), en particulier les $\mathrm{TIF}^{28}$, se développent, permettant de lancer ces grands programmes de renouveau urbain typiques de l'urbanisme néo-libéral de la ville.

L'écart présent dès la construction de la ville entre un centre-ville en plein essor et l'ouest proche s'est ainsi creusé depuis le dernier tiers $\mathrm{du} \mathrm{xx}^{\mathrm{e}}$ siècle $^{29}$. Le premier est à la fois quartier des affaires, des grandes institutions culturelles et des classes 
supérieures tandis que le second est encore en déprise industrielle et lieu de refuge des anciennes populations d'ouvriers noirs et latinos. En conséquence, l'absence de mixité sociale et ethno-raciale - qui contribue à donner un visage ethnique à la pauvreté s'est accentuée dans certains districts de la partie occidentale et du sud, surtout depuis la crise de 2008. C'est notamment le cas du Lower West Side où se situe Pilsen. La racialisation à partir des années 1960 a entraîné ou intensifié le slumlordism ${ }^{30}$, le désinvestissement, le redlining ${ }^{31}$, la restriction des investissements municipaux, aggravant ainsi la situation de ségrégation, la réduction et l'exclusion (Betancur J. et Y. Kim's, 2016: 6; Collectif, 2019 :70). Parallèlement, à partir des années 1970, la désindustrialisation et le déclin des chemins de fer ont affecté les chances d'emploi et de mobilité des résidents qui ont dû se tourner vers des emplois à bas salaires dans l'industrie des services. Ce district cumule ainsi des difficultés socio-économiques en lien avec la population racisée. Selon le recensement de 2010 (cité dans EPA, 2013 : 11), $83 \%$ de la population de Pilsen était hispanique contre $12 \%$ blanche. $45 \%$ de la population est née dans l'illinois, $42 \%$ de la population est née à l'étranger et $28 \%$ de ces derniers ne parlent pas ou mal l'anglais. En 2011, 31 \% de la population était sous le seuil de pauvreté et le revenu médian annuel estimatif par ménage ne dépassait pas 40 553 dollars (alors qu'il était pour la ville de 43628 dollars) ${ }^{32}$.

15 Parallèlement, de grandes friches industrielles se sont libérées dans toute la partie sud, sud-est du district, accentuant la vulnérabilité du quartier aux spéculateurs. À Chicago, la pression foncière sur l'est de la ville étant très forte, un report du marché immobilier au profit des jeunes classes moyennes blanches s'opère vers l'ouest proche. Le Lower West Side, très bien situé, devient une "gentrification frontier" ${ }^{33}$. Des artistes tentent alors l'installation dans ce quartier délabré, encore bon marché, mais en voie de rénovation urbaine ${ }^{34}$.

Plusieurs chantiers vont ensuite contribuer au cours de différents cycles à installer un processus de renouveau urbain, de modification démographique et socio-économique, d'est en ouest du district, avec l'appui de la mairie.

C'est, tout d'abord, la construction de l'extension du campus de la University of Illinois at Chicago (UIC). Commencée sous le mandat de Richard J. Daley ${ }^{35}$, l'université s'étendait en 1982 jusqu'au 750 S. Halsted, dans ce qui était Little Italy. En 2007, un village universitaire avec résidences étudiantes, commerces et soins de santé a été construit, amenant une nouvelle population étudiante dans le East Pilsen ${ }^{36}$.

18 Parallèlement à l'University Village de la UIC, une communauté d'artistes ${ }^{37}$ s'établit dans East Pilsen à la faveur du rachat de vieilles fabriques et de leur transformation par un promoteur du quartier (John Podmajersky fils). Surnommé "Podville», ce nouvel ensemble va continuer à se développer à la génération suivante (avec J. Podmajersky petit-fils qui poursuit les alliances familiales avec le maire Daley fils). A partir de 2002, Podville renaît en tant que "Chicago Art District», renforçant ainsi la stratégie d'utilisation des artistes résidents comme vecteur d'attraction d'un nouveau marketing culturel $^{38}$. Ces pratiques de art washing ${ }^{39}$ s'opèrent à la faveur des nouvelles politiques développées par le Département des affaires culturelles de la ville (Grams, 2010, p. 172). Celles-ci vont se systématiser sous le mandat de Rahm Emmanuel (2011-2019), pendant lequel on insiste sur l'idée que les arts, la culture et le «capital humain » stimulent la croissance ${ }^{40}$.

19 Mais les habitants ont également contribué à rendre plus vivable le quartier.. L'insécurité a gangrené les lieux dans les années 1980 et 1990, puis a connu quelques 
épisodes côté ouest dans les années $2000^{41}$. De nombreuses actions positives ont alors été menées par les résidents et artistes mexicains, les associations éducatives et culturelles, les églises également, pour un vivre-ensemble dans leur quartier ${ }^{42}$. Dans le même temps, les pouvoirs municipaux et d'autres figures politiques emblématiques de l'Etat, tel le sénateur Obama en 2006, ont développé des campagnes pour la coexistence pacifique des communautés dans la ville.

La chute des tensions entre bandes côté occidental, l'installation proche du campus UIC et de Podville à l'Est, ont eu comme effet un remplacement démographique notable ${ }^{43}$. L'est de Pilsen est devenu la plaque tournante des hipsters (bobos, jeunes branchés, artistes, industries créatives) principalement blancs. Un nouveau front, le CentrePilsen, s'ouvre également dans les années 2000 (Betancurt J. et Y. Kim's, 2016) ${ }^{44}$. Les « forces gentrifiantes » se voient un temps ralenties par la crise de 2008, mais les saisies immobilières et les ventes à découvert relancent l'entrée de nouveaux acquéreurs (op. cit., $2016: 7)$.

21 Le processus de gentrification s'opère principalement au profit de la classe moyenne blanche ${ }^{45}$. Une étude récente a montré que les résidents de White Pilsen gagnent en moyenne plus que leurs homologues latino-américains. Leur arrivée a coïncidé avec une hausse du loyer mensuel médian de Pilsen (de 483 dollars en 2000 à 778 dollars en 2013) (Betancur J. et Y. Kim's, 2016). Comme en témoigne C. Bravillo ${ }^{46}$, graveur nicaraguayen et activiste, qui anime des ateliers au Pilsen Outpost, l'un des derniers endroits où l'on enseigne la gravure sociale : «la première chose c'est que l'on remplace les tortillerías, les restaurants mexicains et les associations mexicaines par des bars Lounge comme le Thalia Hall; les artistes latinos sont poussés en dehors de leur atelier et les immeubles de l'architecture bohémio-tchèque [nda : construits entre la fin du XIX siècle et les années 1940] sont démolis pour les remplacer par des condominiums. On remarque tout de suite que la clientèle a changé quand les enseignes en espagnol disparaissent et que les clients qui attendent sur le trottoir sont des "güeros altos y mujeres rubias" (des hommes blancs de haute taille et des femmes blondes) » ${ }^{47}$. Selon Betancur J. et Y. Kim's (2016: 58), la montée en puissance de la culture blanche à Pilsen peut d'ailleurs se constater par la création de deux festivals, l'un mexicain, l'autre blanc.

22 Les habitants mexicains modestes (journaliers, ouvriers, familles de bas revenus) sont poussés hors du quartier. Les artistes locataires doivent abandonner leur atelier face aux augmentations de loyer, comme me l'a confié une jeune artiste originaire de Guadalajara. Du fait des dernières restrictions des lois migratoires, il est extrêmement difficile aux nouveaux arrivants de remplir les conditions d'obtention d'un permis de séjour. Ces locataires sans-papiers sont plus facilement expulsables de leurs logements, n'ayant pas de recours légal contre leur propriétaire. On assiste bien là à l'intensification de formes d'inégalités socio-spatiales qui se cumulent entre elles (situation familiale, origine, pauvreté) et qui provoquent l'éloignement vers des zones de relégation.

D'autres résidents, propriétaires de maisons individuelles dans le district, se voient en difficulté pour entretenir leur logement ou payer les taxes foncières. Ils finissent par vendre aux compagnies immobilières qui rentabilisent la forte densification autorisée par le plan de zonage. Une partie du foncier est en effet classé en R-4, c'est-à-dire permettant de construire, pour une destruction, quatre logements ( $c f$. Curran W. et E. Hague, 2006). 
Les structures assurant la vie culturelle, sociale et communautaire mexicaine souffrent fortement de cette situation et disparaissent de l'espace public, comme c'est le cas de Casa Aztlán. Les peintures murales de la façade ont été recouvertes d'un enduit gris par le nouveau propriétaire. Cette situation est ressentie par les résidents comme une rupture emblématique du lien politique au territoire : «en perdant ces peintures murales, en perdant cet immeuble, on perd la communauté, c'est comme si nous étions tous dispersés $»^{48}$ (illustration 8). C. Taviria ${ }^{49}$, du Musée mexicain de Pilsen (NMMA), considère que ce type d'effacement de la présence d'une communauté dans la ville est la preuve d'un « racisme structurel des Blancs (Whites). La première chose que font les nouveaux propriétaires est d'effacer les peintures murales pour normaliser le quartier $\aleph^{50}$. Cette pratique de whitewashing est caractéristique des processus de gentrification. En visant à effacer les signes distinctifs, elle rend l'espace public neutre pour de nouveaux arrivants ${ }^{51}$.

Illustration 8 : Casa Aztlán - Peintures originales de Ray Patlan

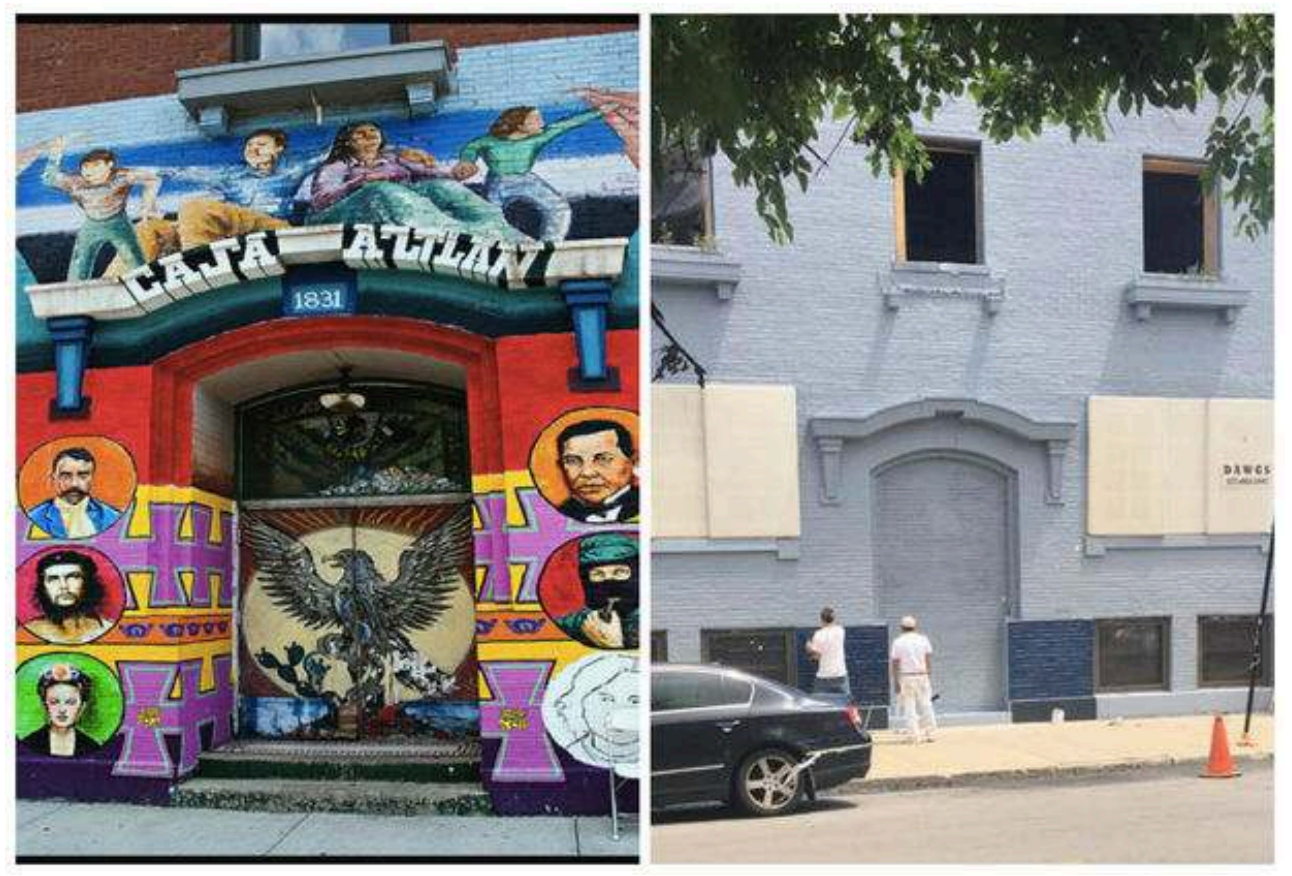

L'un des premiers centres communautaires de Pilsen. La façade a été repeinte en gris par le promoteur (1831 South Racine Avenue).

Crédit : Pilsen Alliance ; Facebook/Ricardo Gamboa

J'ai également pu constater que de nombreux tracts étaient affichés par des collectifs contre la gentrification dans les vitrines des commerces mexicains ${ }^{52}$. La gentrification y est analysée comme le remplacement des résidents par un groupe socio-racial économiquement plus performant, qui efface la diversité culturelle et l'identité de la working class. Il s'agit là de représentations caractéristiques des processus de gentrification qui font émerger une dimension intersectionnelle entre race et classe.

Devant cette situation, l'élection de Lori Lightfoot à la mairie de la ville depuis le 2 avril 2019 rebattra-t-elle les cartes? La nouvelle mairesse - africaine-américaine et homosexuelle revendiquée - a en effet fondé sa campagne politique sur l'appui aux communautés racisées et LGBTQI+. Un changement pourrait-il alors se dessiner en développant une politique urbaine et culturelle plus inclusive, répondant aux besoins 
des populations vulnérables et des quartiers ségrégés ? Le rapport de transition élaboré par 400 citoyens éminents représentant les différentes communautés de la ville et remis à L. Lightfoot va dans ce sens (Collectif, $2019: 28)$. Une série de recommandations de l'Arts and Culture committee relève la difficulté de conserver des artistes locaux ainsi que des actions artistiques dans les quartiers (neighborhoods), si les politiques publiques ne pensent pas de manière simultanée un certain nombre de mesures. Il faut notamment "financer la rénovation des bâtiments abandonnés, en priorisant l' utilisation artistique en fonction des besoins exprimés par la communauté » (ibid.). Dans le même temps, on doit penser l'accès au logement, sa réhabilitation, la mise en place de mécanismes pour assurer des loyers abordables, le tout associé au développement du quartier (éducation, sécurité, connexion aux transports) (ibid.). Il s'agit bien là d'articuler la tension entre la gentrification, l'expulsion des populations à faibles revenus et la possibilité de maintenir des pratiques culturelles habitantes liées à la culture de la communauté migrante.

\section{Gentrification, cultures et classe à Pilsen}

Cette unanimité dans les protestations et les slogans occulte toutefois la complexité des mécanismes de ce qu'on pourrait appeler «les gentrifications »- selon la formulation de Chabrol M. et al. (2016) - et de ses effets. Les données recueillies en mai et juin 2020 lors d'une enquête en porte-à-porte auprès des commerçants et résidents de la calle dieciocho (18th Street), m'ont ainsi permis de mieux analyser les intérêts contradictoires et les lignes de fracture au sein de la communauté mexicaine.

En juin 2018, Forbes a classé Pilsen comme " one of the "12 Coolest Neighborhoods Around the World" ", célébrant ses galeries, ses peintures murales colorées, ses artistes locaux et l'art de vivre à la mexicaine (gastronomie, musique, fête, art et culture) ${ }^{53}$. Haute culture mexicaine, pratiques culturelles habitantes, patrimoine culturel migrant confondus constituent des ressources matérielles et symboliques dont les magazines, tel Forbes, se font le relais. Cette marchandisation de la culture mexicaine - le Mexican branding - revient à faire du Pilsen mexicain « un site plus viable de «consommation ethnique ", en particulier par sa culture festive » (Anderson M. et C. Sternberg, 2013 : 452). Requalifiées à travers une chaîne d'intermédiaires et de mises en récit, ces ressources permettent de construire une économie marchande, qui profite notamment au secteur immobilier financiarisé, pour créer et vendre de nouveaux espaces urbains. Le gouvernement local municipal et infra-municipal (district) est central dans ce dispositif. Il permet de stimuler par des politiques urbaines et culturelles l'idéologie, les valeurs et les outils permettant de créer une structure d'opportunité. Les «forces gentrifiantes » ont ainsi fait de la culture le point-clé de la vente du réaménagement des quartiers abritant des minorités à forte valeur culturelle ajoutée ${ }^{54}$.

Comme le rappelle le sociologue S. Chauvin (2014), Chicago est la ville fière de ses quartiers et en même temps, le lieu d'une ségrégation raciale unique dans le pays : « Le gouvernement néolibéral de la cité [est] fondé sur la célébration des différences culturelles associées à des espaces délimités [...Il] a fait de la race (mais aussi du territoire) la 'modalité selon laquelle la classe est vécue" " 55 . Ces "espaces délimités " fondés sur le capitalisme de célébration de la différence constituent par là-même une stratégie de revitalisation des vieux quartiers ethniques. 

Selon C. Taviria : «Ce classement de Forbes est le signe que la ruée vers le quartier est ouverte. On pousse dehors les Mexicains. La première chose que l'on fait c'est d'effacer les murales des façades, mais ils ne pourront pas totalement nous mettre dehors, il y aura toujours une présence mexicaine dans ce quartier, même si ce n'est que de 30 à $40 \%$. Ma fille, par exemple, a décidé d'acheter un condominio ici à Pilsen, et d'autres sont en train de le faire $\Perp^{56}$. C. Taviria fait ici allusion à une situation que j'ai pu constater lors de mon enquête de mai-juin 2019. Comme les jeunes classes moyennes blanches, des Mexicains aux revenus médians, parfois des Mexicains-Américains de la deuxième génération, ont ainsi décidé de réinvestir « leur " quartier, devenu plus sûr. Des immigrants arrivés dans les années 1980 du Sud-Ouest mexicain (Etat de Guerrero) et ayant "réussi », tout comme des Mexicains-Américains de Californie, ont ainsi acheté les commerces d'artisanat de la calle 18. Une petite fraction d'entre eux profite $\mathrm{du}$ boom des loyers, de la hausse des prix et de la mise en tourisme du quartier. Cela leur assure une clientèle extérieure à meilleurs revenus, désireuse d'acheter des produits d'artisanat d'art et de visiter le National Museum of Mexicain Art, devenu très à la mode. La même démarche de Mexican Branding s'observe dans de nouvelles galeries et des lieux culinaires «à la mexicaine » (mais pas pour les Mexicains). Ces propriétaires ont aussi investi dans le locatif et ne se montrent pas nécessairement de meilleure composition envers leurs locataires que les bailleurs d'autres origines. concomitante aux changements de règlements municipaux qui, par des taxes différentielles selon le quartier, tendent à accélérer les mécanismes de mobilité des populations $s^{57}$. Quelques professionnels latinos de l'immobilier appuient le processus visant à étendre la frontière de la gentrification jusqu'à l'Ouest de Pilsen. Betancur J. et Y. Kim's (2016: 8) soulignent les connexions politiques de ces acteurs, de même que la position pro-gentrification du conseiller municipal (alderman) de Pilsen - d'origine mexicaine - soutien du maire Daley fils dans le district et qui a exercé de 1996 à 2019. Les interactions des pouvoirs municipaux avec les promoteurs immobiliers et les acteurs des marchés financiers, en lien avec les acteurs locaux (élus de district, certaines organisations communautaires pour l'habitat abordable) conditionne de manière systémique la production du renouveau urbain. Elles organisent les changements de zoning (densification et changement d'usage des sols) et captent le surplus des différentes taxes, ce que certains ont appelé la «machine Dailey » (Weber R., 2015).

Il est également notable que certains des Mexicains et Mexicains-Américains les mieux intégrés se réjouissent d'un nouvel apport de population mexicaine plus aisée pour leur quartier. Ils considèrent que cela permettra à la raza et à la culture mexicaine de continuer à vivre à West Pilsen. De cette façon, le quartier représenterait l'image de la réussite économique mexicaine, de ses forces créatives, de ses managers et entrepreneurs dans la ville. Des exemples de trajectoire sociale réussie m'ont été citées à l'appui, comme la première femme d'origine mexicaine dirigeante de McDonald's Illinois, également donatrice et membre du conseil d'administration du Musée d'art mexicain ${ }^{58}$. Cette alliance de l'ascension économique - un latino capitalism ? - et de la reconnaissance par le milieu culturel, ne serait-ce pas là le rêve américain de ces acteurs sociaux ${ }^{59} \mathrm{Comment}$ doit-on considérer ces faits si l'on se réfère aux modèles existants sur la gentrification? Certains auteurs parlent d'une gentrification « Latino- 
on-Latino" du fait que des Mexicains de revenu intermédiaire participent au mouvement de changement urbain (Anderson M. et C. Sternberg, 2013 ; Badger E., 2012). D'autres préfèrent parler de gentrification white-on-latino, en rappelant que les «forces gentrifiantes » sont majoritairement blanches à Pilsen, même si elles incluent des gentrifieurs mexicains et mexicains-américains. Une petite partie de Mexicains à bas revenus a, il est vrai, réussi jusqu'à présent à se maintenir sur place (Betancur J. et Y. Kim's, 2016 : 19, 59). Sur ce dernier point, un front de résistance, le Pilsen Land Use Committee (PLUC), s'est formé à partir de différentes associations de résidents. Au moyen d'un referendum positif mené auprès de la population en 2005, le PLUC a réussi à s'imposer comme instance consultative auprès de l'alderman avec le principe d'un quota de $21 \%$ de logements à prix abordable pour toute construction nouvelle (plus élevé que les 10-12 \% généralement prévus) (Curran W. et E. Hague, 2006).

\section{Conclusion}

Pour conclure, le cas de Pilsen permet d'analyser les effets paradoxaux de la pacification urbaine, des politiques identitaires, mais aussi de l'investissement dans la culture et les arts dans un quartier d'immigration mexicaine. Quand la culture en ville est instrumentalisée au profit de l'attractivité territoriale, elle facilite, de fait, les processus de gentrification.

Par ailleurs, faut-il le rappeler, l'organisation sociale de l'espace urbain est productrice d'injustice mais aussi d'opportunités, selon le statut, les revenus et les intérêts de classe des groupes racialisés dans la ville. Les actions menées par les artistes locaux et les habitants, à travers la culture, montrent ainsi le champ de résistance, « d'empouvoirement» (empowerment) créé, leur capacité d'auto-organisation et de construction de collectifs. Face à des questions - la gentrification, la culture locale migrante vs les Blancs, la classe, la relégation - qui rapprochent et à la fois divisent les agents, ces collectifs arrivent dans certaines étapes des luttes à un consensus d'actions pour une meilleure égalité dans la ville ${ }^{60}$.

Il restera à observer les alliances futures possibles entre East Pilsen et West Pilsen, entre le White Pilsen et le Mexican Pilsen de classe moyenne, au-delà de la marchandisation des cultures ethniques et de l'art washing des gentrifieurs et de leurs relais publics.

\section{BIBLIOGRAPHIE}

Abu-Lughod, Janet L., Race, Space and Riots, in Chicago, New York and Los Angeles, New York, Oxford University Press, 2007.

Anderson, Mathew B. \& Carolina Sternberg, « "Non-White” Gentrification in Chicago's Bronzeville and Pilsen Racial Economy and the Intraurban Contingency of Urban Redevelopment ", Urban Affairs Review, 49 (3), 2013, p. 435-467. 
Arango Juárez, Carlos, Omar López Zacarías et Jorge Mújica Murias, Voces migrantes. Movimiento 10 de marzo, Chicago, El BeisMan Press, 2016.

Arredondo, Gabriela F., Mexican Chicago: Race, Identity, and Nation, 1916-39. Becoming Mexican in early twentieth-century Chicago, Chicago, University of Illinois Press, 2008.

Badger, Emily, « How Black Gentrifiers Have Affected the Perception of Chicago's Changing Neighborhoods ", Citylab, 31 décembre 2012, http://www.citylab.com/housing/2012/12/howBlack-gentrifiers-have-affected-perception-chicagos-changing- neighborhoods/4233/, page consultée le 8 janvier 2021.

Betancur, John J. \& Youngjun Kim's, The Trajectory and Impact of Ongoing Gentrification in Pilsen, Nathalie P. Voorhees Center for Neighborhood and Community Improvement (the Voorhees Center) at the University of Illinois at Chicago (UIC), 2016, https://voorheescenter.uic.edu/ reports/, page consultée le 6 janvier 2021.

Biles, Roger, Richard J. Daley: Politics, Race, and the Governing of Chicago, DeKalb, IL., Northern Illinois University Press, 1995.

Boyd, Michelle, "Reconstructing Bronzeville: Racial Nostalgia and Neighborhood Redevelopment", in Journal of Urban Affairs, April, 22 (2), 2000, p. 107-122.

Certeau, Michel (de), L'Invention du quotidien, 1 : Arts de faire, éd. établie et présentée par Luce Giard, Paris, Gallimard, 1980.

Chabrol, Marie, Anaïs Collet, Matthieu Giroud et al., Gentrifications, Paris, Ed. Amsterdam, 2016. Chauvin, Sébastien, « Chicago et l'exception urbaine américaine », La Vie des idées, 15 décembre 2014, https://laviedesidees.fr/Chicago-et-l-exception-urbaine.html, page consultée le 2 janvier 2021.

Collectif, The Chicago Cultural Plan, 1986, https://web.archive.org/web/20111226044349/http:// www.cityofchicago.org/content/dam/city/depts/dca/general/ChicagoCulturalPlan.pdf, page consulté le 08/09/2020.

Collectif, Report of the Transition Commitee, submitted to Mayor-elect Lori E. Lightfoot, Chicago, May 17, 2019, https://bettertogetherchicago.com/transition-report/, page consultée le 05/09/2020.

Curran, Winifred \& Euan Hague, The Pilsen Building Inventory Project, DePaul University, Department of Geography, 2006, https://www.academia.edu/25625357/ The_Pilsen_Building_Inventory_Project page consultée le 10 février 2021.

Curran, Winifred, Gender and Gentrification, Critical Studies in Urbanism and the City, Routledge, Oxon and New York, 2019.

Denton, Nancy A. et Douglas S. Massey, American apartheid, Paris, éd. Descartes et Cie, 1995 [1992]. Diamond, Andrew et Caroline Rolland-Diamond, « Au-delà du Vietnam : Chicago 1968 et l'autre mouvement étudiant-lycéen », Histoire@Politique. Politique, culture, société, n6, septembredécembre 2008, www.histoire-politique.fr, page consultée le 10 décembre 2020 Diamond, Andrew J., « Chicago : une ville au bord du gouffre », La Vie des idées, 29 mai 2012, https://laviedesidees.fr/Chicago-une-ville-au-bord-du-gouffre.html page consultée le 10 décembre 2020.

EPA (The U.S. Environmental Protection Agency), Community Involvement Plan for the Pilsen and Little Village Sites Chicago, Illinois, s.l., 2013. 
Florida, Richard, Cities and the Creative Class, New York, London, Routledge, 2005.

Gervais, Laurence, Le sexe de la ville : identités, genre et sexualités dans la ville états-unienne, Paris, Syllepse, 2020.

Glaser, Barney G. and Anselm L. Strauss, The Discovery of Grounded Theory: Strategies for Qualitative Research, Chicago, Aldine Publishing Company, 1967.

Grams, Diane, Producing Local Color: Art Networks in Ethnic Chicago, Chicago, The University of Chicago Press, 2010.

Hall, Stuart, « New Ethnicities », in David Morley et Kuang-Hsing Chen (dir.), Stuart Hall : Critical Dialogues in Cultural Studies, Londres et New York, Routledge, 1996, p. 441-449.

Hémond, Aline, « Habiter par l'art : migration mexicaine et art muraliste social à Chicago, Pilsen », in La part artistique de l'habiter : perspectives contemporaines, Olivier Lazzarotti, Guy Mercier, Suzanne Paquet (eds.), L'Harmattan, coll. « Géographie et cultures », 2017, p.129-155. Huante, Alfredo, « A Lighter shade of Brown? Racial Formation and Gentrification in Latino Los Angeles », Social Problems, spz047, 68 (1), February 2019, p. 63-792.

Kwon, Miwon, One place after another: site-specific art and locational identity, Cambridge, Mass, MIT Press, 2002.

Lees, Loretta, Tom Slater \& Elvin Wyly, Gentrification, New York, Routledge, 2008.

Lefebvre, Henri, « Le droit à la ville », L’homme et la société, n 6, 1967, p. 29-35.

Ley, David, The New Middle Class and the Remaking of the Central City, Oxford, Oxford University Press, 1996.

Luke, David J., « Redlining », in Encyclopedia of Human Services and Diversity, Linwood H. Cousins (ed.), SAGE Publications, Inc., Thousand Oaks, 2014, http://dx.doi.org/ 10.4135/9781483346663.n480, page consultée le 10 janvier 2020.

Lussault, Michel, «Visibilité (régime de) », in Jacques Lévy et Michel Lussault (éd.), Dictionnaire de la géographie et de l'espace des sociétés, Paris, Belin, 2003, p. 997.

Mirabal, Nancy Raquel, « Geographies of Displacement: Latina/os, Oral History, and The Politics of Gentrification in San Francisco's Mission District », The Public Historian, 31 (2), 2009, p. 7-31.

O'Brien, Kean, Leonardo Vilchis et Corina Maritescu, « Boyle Heights and the Fight against Gentrification as State Violence », American Quaterly, 71 (2), 2019, p. 389-396.

Olivier De Sardan, Jean-Pierre, La rigueur du qualitatif. Les contraintes empiriques de l'interprétation socio-anthropologique, Louvain-la-Neuve, Academia Bruylant, 2008.

Pattillo, Mary, Black on the Block: The Politics of Race and Class in the City, Chicago, The University of Chicago Press, 2007.

Ramírez, Leonard G., Yenelli Flores, Maria Gamboa \& al., Chicanas of $18^{\text {th }}$ Street. Narratives of a Movement from Latino Chicago (Latinos in Chicago and Midwest), Urbana, Chicago and Springfield, University of Illinois Press, 2011.

Rodríguez, Marc, « Latino Mural Cityscapes: A Reflection on Public Art, History, and Community in Chicago after World War II. » in EthniCities: Metropolitan Cultures and Ethnic Identities in the Americas, Martin Butler, Jens Martin Gurr, Olaf Kaltmeier (ed.), Bilingual Press, Tempe Arizona, 2011, p. 67-77. 
Rogovin, Mark, Marie Burton \& Holly Highfill, Mural Manual. How to paint Murals for the Classroom, Community Center and Street Corner, edited by Tim Drescher, Boston, Beacon Press, 1975.

Sachs Olsen, Cecilie, Socially Engaged Art and the Neoliberal City, New York, Routledge, 2019.

Smethurst, James, The Black Arts Movement: Literary Nationalism In The 1960s And 1970s, Chapel Hill, The University of North Carolina Press, 2005.

Smith Neil, The New urban frontier: gentrification and the revanchist city, New York, Routledge, 1996.

Smith, Neil, « Toward a Theory of Gentrification: A Back to the City Movement by Capital, not People », Journal of the American Planning Association, vol. 45, 1979.

Soja, Edward W., «La ville et la justice spatiale ", Justice spatiale/Spatial Justice, $\mathrm{n}^{\circ}$ 1, septembre, 2009, https://www.jssj.org/article/la-ville-et-la-justice-spatiale/, page consultée le 10 janvier 2021.

Weber, Rachel, From Boom to Bubble. How Finance built the New Chicago. Chicago, University of Chicago Press, 2015.

Zukin, Sharon, Loft Living: Culture and Capital in Urban Change, Baltimore, Johns Hopkins University Press, 1982.

\section{NOTES}

1. Je remercie vivement mes relecteurs pour leur contribution analytique et leurs conseils bibliographiques.

2. Sur l'art social engagé, voir Sachs Olsen C. (2019); Hémond A. sur Pilsen (2017).

3. Boyd M. (2000) parle de « tourisme du patrimoine racial » (racial heritage tourism), en partant de l'exemple du quartier africain-américain de Bronzeville, au sud de Chicago.

4. A ce propos, consulter Curran W. (2019).

5. L'explication par la demande "se place du côté de la demande de logements et de services et explique la gentrification par les goûts d'une nouvelle classe sociale urbaine désireuse de vivre en centre-ville» (Chabrol M. et al., $2016: 27$ ). Elle met l'accent sur la consommation et la culture (Ley D., 1996). Voir aussi les travaux de Lees L. et al. (2008).

6. L'explication de la gentrification par l'offre a été portée par les marxistes structuralistes tel le géographe N. Smith (1979) qui mettent l'accent sur la production et le rôle du capital.

7. Sur les intérêts de classe au sein du même groupe racialisé, voir le travail de Pattillo M. (2007) sur la gentrification du quartier africain-américain de North Kenwood-Oakland au nord de Chicago.

8. Par point de vue emic, je me réfère aux courants anthropologiques qui privilégient le point de vue indigène ou populaire et le contrastent avec l'etic ou point de vue savant (cf. Olivier de Sardan J.-P., 2008). Pour ce faire, je pars de l'ethnographie des acteurs sociaux et du sens local qu'ils donnent aux faits avant de faire émerger une théorie ancrée sur le terrain (ou grounded-theory : Glaser B. et Strauss A., 1967). Les données ethnographiques utilisées pour cet article ont principalement été recueillies en mai et juin 2019.

9. Le Chicano Movement est un mouvement de résistance politique, social et culturel visant à redonner une importance et une légitimité aux Mexicains des Etats-Unis en affirmant une culture propre. De nombreuses figures importantes, syndicales, littéraires et artistiques ont donné leurs lettres de noblesse au Chicano Art (avec le mythe du retour à Aztlán par exemple).

10. Discussions et entretiens avec Carlos Arango (juin 2019), Nicolás de Jesús (2014, 2016), Franky Piña (mai 2019). Observation des défilés des organisations contre les lois migratoires (Memorial Day, 27 mai 2019). 
11. Les Mexicains s'autodénominent entre eux La raza (litt. « mon peuple »), en contraste avec les exonymes administratifs hispano ou latino.

12. La formulation est mienne.

13. La fresque "A la esperanza » était un projet de la Casa Aztlán qui fut sélectionné par les habitants. Il a été conçu par Malú Ortega et Jimmy Longoria, assisté par les artistes Salvador Vega, Marcos Raya, et Oscar Moya. Une exposition au National Museum of Mexican Art commémore ces mobilisations de la communauté mexicaine de Pilsen. http:// nationalmuseumofmexicanart.org/exhibits/40-a\%C3\%B1os-la-esperanza.

14. Le Wall of Respect, dirigé par William Walker, a été peint dans le ghetto de Bronzeville, au sud de Chicago, par des artistes du groupe OBAC qui participent au Black Arts Movement (BAM). Cette œuvre a été à l'origine d'un mouvement de création de peintures murales communautaires dans de nombreuses villes américaines (Smethurst J., 2005).

15. En 1968, Mario Castillo crée Metaphysics (Peace), généralement considérée comme la première peinture murale dans l'espace public du mouvement Chicano aux Etats-Unis ( $c f$. Rodríguez M., $2011: 68)$.

16. Parmi les muralistes les plus célèbres, citons Dr Atl, Orozco, Alfaro Siqueiros et Rivera.

17. Entretiens et histoires de vie recueillis auprès d'artistes et participants de cette époque : Héctor Dúarte, René H. Arceo, Nicolás de Jesús, Carlos Tortolero, Carlos Arango, Mark Rogovin, Winifred Godfrey, William Goldmann, Marcos Raya, Carlos Berberena, Alfredo Mártinez, Erasmo Salgado. Voir également Rodriguez (2011).

18. Notion théorisée par M. Lussault (2003, p. 997).

19. Henri Lefebvre (1967) définit le droit à la ville, comme un droit de base, constitutif de la démocratie, et les villes comme des biens communs accessibles à tous les habitants. A sa suite, le courant de pensée autour de la justice spatiale (Soya E., 2009) va se centrer sur les inégalités socio-spatiales urbaines.

20. Du 16 août 2019 au 15 mars 2020.

21. Voir Ramírez L. \& al., (2011), Chicanas of 18th Street.

22. Entretien avec Carlos Arango, dernier responsable de Casa Aztlán (juin 2019).

23. Sur les manifestations de 2006, voir Arango Juárez C. et al. (2016).

24. Voir le site du Musée : http://nationalmuseumofmexicanart.org/.

25. Sur cet épisode, voir plus bas dans le texte.

26. Avec un succès certain, puisque l'économie culturelle et touristique, à partir de 2007, va générer plus de 11 milliards de dollars par an dépensés par les touristes nationaux et internationaux (cf. Travel Industry Association of America, Direct Impact of Travel to Chicago, in Diamond A., 2012).

27. La rédaction d'un Chicago Cultural Plan en 1986 sous son mandat, qui analyse les besoins et les possibilités culturelles de la ville, affirme que «Culture matters» (Collectif, $1986: 6$ ), c'est-à-dire que la vitalité culturelle est importante pour l'économie et le développement communautaire.

28. C'est notamment le programme de Tax increment Financing (TIF) qui, avec le surplus estimatif des taxes résidentielles, abonde un fonds public-privé qui permet de réaliser différents travaux de "redéveloppement urbain" dans les districts délaissés diagnostiqués comme prioritaires (dont deux projets à Pilsen). Les TIF ont été vivement critiqués par leur gestion très opaque (Weber R., 2015).

29. Chicago est un cas emblématique de la ville segrégée nord-américaine, cela depuis sa création (Denton N. et D. Massey, 1995 [1992]).

30. Il s'agit de marchands de sommeils, propriétaires peu scrupuleux qui maximisent leurs loyers sans investir dans la réfection de leurs biens.

31. Le redlining provient d'une pratique des banques d'entourer certaines zones en rouge sur une carte et de refuser tout prêt hypothécaire pour des biens à l'intérieur de la zone considérée. Le terme a été popularisé dans les années 1960 par le sociologue John McKnight et la pratique 
interdite par la loi de 1968. Cette dernière interdit la discrimination à la vente, à la location de logements fondée sur la race, la religion, l'origine nationale et, depuis 1974, sur le sexe (Luke D., 2014).

32. Données démographiques de la ville (citées dans EPA, 2013 :11).

33. Selon l'expression de Neil Smith (1996:186-187, cité dans Chabrol M. et al., 2016 : 39).

34. Ce processus, bien connu, a été analysé par Sharon Zukin (1982) dans son travail sur la conversion des friches industrielles en lofts du quartier de Soho, à New York, par des artistes à la recherche de lieux spacieux et bons marchés (in Chabrol M. et al., $2016: 32$ ). Si l'on considère la gentrification comme un processus graduel de long terme de mise en valeur attirant les capitaux (dans la lignée de N. Smith, 1996), les artistes tiendraient le rôle, nolens volens, du «corps expéditionnaire des gentrifieurs du centre-ville » - selon l'expression de D. Ley (1996) - avant de subir leur éviction progressive (ibid.)

35. Selon Biles R. (1995:77), ce chantier aurait ainsi permis de freiner l'expansion des populations noires et latinos vers le Loop en stabilisant une zone de valeurs immobilières élevées (in Diamond A. et C. Rolland-Diamond, $2008: 10$ ).

36. Pour ce cas, il serait probablement plus opportun de parler de "studentification" que de gentrification (Betancur J. et Y. Kim's, 2016 : 40). Sur la gentrification de East Pilsen, voir Curran W. et E. Hague (2006).

37. Beaucoup de ces artistes ont d'ailleurs été déplacés par la rénovation urbaine de Hyde Park dans les années 1960.

38. Sur Podville et les conséquences de la gentrification pour les réseaux artistiques locaux à East Pilsen, consulter D. Grams (2010, chap. VII).

39. Voir également le cas de Boyle Heights en Californie (O’Brien K. et al., 2019).

40. A ce sujet, voir Richard Florida (2005) et sa thèse de la Creative class (ou des talents, pour reprendre ses termes) en tant que facteur déterminant du développement économique en milieu urbain. Cette thèse a largement été reprise par les politiques de revitalisation des villes étatsuniennes.

41. En 2017, le système de données de la ville signalait un indice du crime de 468,8 contre la moyenne américaine de 218,3 (http://www.city-data.com/neighborhood/Lower-West-SideChicago-IL.html).

42. Voir le projet muraliste pour l'éducation au vivre-ensemble entre enfants mexicains et africains-américains du voisinage : le J-Def Peace Project https://thejdefpeaceproject.weebly.com/. 43. Le déclin des familles $(20,4 \%)$ et des familles avec enfants de moins de 18 ans (40,9\%), jumelé à l'augmentation des ménages d'une personne $(17,8 \%)$ et des ménages non familiaux $(88,6 \%)$, montre la gentrification opérante entre 2000 et 2010 à Pilsen (Betancur J. et Y. Kim's, $2016: 13$ ).

44. Signalons également l'installation de la communauté lesbienne établie jusque -là à Andersonville (nord-est de la ville), chassée par l'augmentation des loyers et du foncier (Gervais L., 2020).

45. En termes de changement de population, près d'un quart de la population hispanique est partie (9500 personnes) entre 2000 et 2010 - soit $25 \%$ de la population - et 1175 blancs non hispaniques se sont installés. Pour East Pilsen, cette installation blanche a eu lieu dès 1990-2000.

46. Le nom a été changé.

47. Entretien personnel, juin 2019.

48. On peut consulter la vidéo à https://news.wttw.com/2017/07/06/loss-iconic-pilsen-muralsparks-outrage.

49. Le nom a été changé.

50. Entretien personnel, sept. 2020.

51. C'est notamment le cas en Californie. Voir par exemple Huante A., 2019 ; O'Brien K. et al., 2019 ; Mirabal N., 2009. 
52. Notamment le Protect Pilsen Coalition (PPC) mené par C. Arango et J. Valasquez. Mentionnons aussi les actions de Pilsen Alliance, une organisation issue du secteur (grassroot), refusant les partenariats publics-privés. Elle est conseillée par les chercheurs géographes de DePaul University (voir Curran W. et E. Hague, 2006).

53. https://www.forbes.com/sites/annabel/2018/06/22/the-12-coolest-neighborhoods-aroundthe-world/\#7ea99a286eb1

54. Anderson et Sternberg (2013) estiment que la question raciale sous-tend également le choix de la culture à gentrifier, la culture noire de Bronzeville étant moins attractive commercialement que le Pilsen mexicain du fait de préjugés raciaux plus ancrés.

55. Selon l'expression de Stuart Hall (1996:441-449, cité dans Chauvin S., ibid.).

56. Entretien personnel (sept. 2020).

57. L'utilisation des Business Improvement Districts (BIDs) est un autre mécanisme public-privé permettant aux propriétaires de s'associer pour améliorer les services urbains au moyen d'une surtaxe prélevée sur la valeur du foncier de leur périmètre.

58. Il s'agit là de tout un symbole. La société McDonald's actuelle a vu le jour en 1955 à Des Plaines, une banlieue de Chicago. L'entreprise fait l'objet d'une grande fierté de la part des habitants de la ville.

59. Des formes de gentrification non-blanche ont également été montées pour le Black Bronzeville de Chicago (Badger E., 2012; Anderson M. et C. Sternberg, 2013). Les tenants du courant d'analyse autour du Black capitalism considèrent que la réduction des inégalités raciales doit nécessairement passer par le développement de l'entreprenariat et de l'auto-suffisance économique noirs. Cependant, analysé d'une façon critique, ce discours revient à considérer que le Black capitalism offre aux Noirs pauvres la consolation d'être exploités par des Noirs riches plutôt que par des Blancs riches.

60. Dans ses travaux sur la justice spatiale, Soja (2009) a montré l'importance des mécanismes de « coalition » des organisations communautaires de travailleurs pauvres en 1992 à Los Angeles. Ces solidarités nouvelles se jouant entre mouvements aux identités et affiliations multiples ont permis de contrer les processus d'inégalités socio-spatiaux.

\section{RÉSUMÉS}

Ce texte analyse le cas d'un quartier d'accueil des migrants mexicains dans la ville de Chicago (Pilsen), qui abrite nombre d'artistes. Il s'agit de démontrer qu'il existe une tension entre, d'une part, les pratiques culturelles des artistes locaux et, d'autre part, les politiques culturelles et de rénovation urbaine qui visent à assurer l'attractivité territoriale métropolitaine. À travers une ethnographie des acteurs sociaux et artistiques menée en grande partie en 2019, sont d'abord étudiées les pratiques communautaires des artistes immigrants, en particulier dans l'art muraliste. Puis, l'analyse porte sur le processus accéléré de gentrification qui touche le district. Devenu très à la mode du fait de sa rénovation post-industrielle, il attire nouveaux habitants et tourisme des quartiers dits ethniques. Cette dynamique de gentrification organise simultanément la relégation des populations mexicaines les plus vulnérables et la marchandisation $\mathrm{du}$ patrimoine culturel migrant. Enfin, la recherche démontre que l'organisation sociale de l'espace urbain est productrice d'injustice mais aussi d'opportunités, selon le statut, les revenus et les intérêts de classe des groupes racialisés dans la ville. 
This paper analyses the case of a Mexican migrants neighborhood in the city of Chicago (Pilsen) which is home to many artists. The aim is to show that there is a tension between, on the one hand, the cultural practices of local artists and, on the other hand, the cultural policies and urban renewal that aim to ensure a metropolitan territorial attractiveness. Through an ethnography of social and artistic actors mostly conducted in 2019, this piece proposes first to study the community practices of immigrant artists, especially in muralist art. Then the analysis focuses on the accelerated gentrification process in this district. It became very fashionable due to its post-industrial renovation, attracting new inhabitants and tourism of so-called ethnic neighbourhood. This dynamic gentrification simultaneously organizes the displacement of the most vulnerable Mexican populations and the commodification of migrant cultural heritage. Finally, this research shows that the social organization of urban space is not only a source of injustice but also of opportunities depending on the status, income and class interests of racialized groups in the city.

En este texto, se analiza el caso de un barrio de llegada de migrantes mexicanos en la ciudad de Chicago (Pilsen), que alberga a numerosos artistas. Se intentará mostrar que existe una tensión entre, por una parte, las prácticas culturales de los artistas locales $\mathrm{y}$, por otra, las políticas culturales y de renovación urbana destinadas a garantizar el atractivo territorial metropolitano. A través de la etnografía de los actores sociales y artísticos llevada a cabo mayoritariamente en 2019, se estudian las prácticas comunitarias de los artistas inmigrantes, en particular en el muralismo. Luego se enfoca en el proceso acelerado de gentrificación que afecta al distrito. Se ha vuelto muy de moda debido a su renovación postindustrial y atrae a nuevos habitantes y al turismo de los llamados barrios étnicos. Esta dinámica de gentrificación organiza simultáneamente el desplazamiento de las poblaciones mexicanas más vulnerables y la comodificación del patrimonio cultural migrante. Para terminar, la investigación demuestra que el modo en qué la organización social del espacio urbano produce injusticia, pero también oportunidades, según el estatuto, los ingresos y los intereses de clase de los grupos racializados en la ciudad.

\section{INDEX}

Palabras claves : Pilsen, Chicago, artistas mexicanos, gentrificación, Mexican Branding, art washing

Mots-clés : Pilsen, Chicago, artistes mexicains, gentrification, Mexican Branding, art washing

Keywords : Pilsen, Chicago, Mexican artists, gentrification, Mexican Branding, art washing

\section{AUTEUR}

\section{ALINE HÉMOND}

UR UPJV 4287 « Habiter le monde » / Université de Picardie-Jules Verne UFR de Sciences Humaines, Sociales et Philosophie, Département de Sociologie, Ethnologie, Démographie Bureau H113

La Citadelle 10 rue des Français libres - 80080 AMIENS aline.hemond@u-picardie.fr 